\title{
Biologically Inspired Rate Control of Chaos
}

\author{
Tjeerd V. olde Scheper* \\ School of Engineering, Computing and Mathematics, Oxford Brookes University, United Kingdom
}

(Dated: September 18, 2017)

The overall intention of chaotic control is to eliminate chaos, and to force the system to become stable in the classical sense. In this paper, I demonstrate a more subtle method that does not eliminate all traces of chaotic behaviour; yet it consistently, and reliably, can provide control as intended. The Rate Control of Chaos (RCC) method is derived from metabolic control processes, and has several remarkable properties. RCC can control complex systems continuously, and unsupervised, it can also maintain control across bifurcations, and in the presence of significant systemic noise. Specifically, I show that RCC can control a typical set of chaotic models, including the 3 and 4 dimensional chaotic Lorenz systems, in all modes. Furthermore, it is capable of controlling spatiotemporal chaos without supervision, and maintains control of the system across bifurcations. This property of RCC allows a dynamic system to operate in parameter spaces that are difficult to control otherwise. This may be particularly interesting for the control of forced systems, or dynamic systems that are chaotically perturbed. These control properties of RCC are applicable to a range of dynamic systems, thereby appearing to have far-reaching effects beyond just controlling chaos. RCC may also point to the existence of a biochemical control function of an enzyme, to stabilise the dynamics of the reaction cascade.

* tvolde-scheper@brookes.ac.uk 


\section{LEAD PARAGRAPH}

The Rate Control of Chaos method is derived from the concepts used in biological metabolic control. The control method allows the stabilisation of chaotic systems into stable states. It is capable of controlling the dynamics of known chaotic model systems, but can also be used to control chaotic systems that are unstable due to spatiotemporal interactions. It can maintain control even when parameter changes cause a change in the dynamics of the system. The method is based on the current state of the chaotic system, and does not require supervision to be effective. It is postulated that it may underpin the basic principles of enzyme mediated reaction control.

\section{INTRODUCTION}

In general, it can be said that the aim of controlling chaotic dynamic systems is to stabilise specific points, or periodic orbits, within phase space. The chaotic nature of a system can thus be reduced to stable states. Different methods have been developed that are variations on the OGY [1,2] control or delay control [3-5]. The OGY control methods require knowledge of the unstable periodic orbits (UPOs) contained in the attractor. Therefore, an analytical understanding of the chaotic system is necessary to control the system. The delay control method uses the control function $F(y)=K(y(t)-y(t-\tau))$ which does not require any knowledge of the UPOs but it needs appropriate choices for the control constant $K$ and the delay $\tau$. If the choices for $K$ and $\tau$ are not correctly chosen, then the system will not stabilise into an orbit. Note that some chaotic systems cannot be stabilised using the single delay control method, such as the three dimensional Lorenz system, but these can be controlled by an extended delayed feedback control $[5,6]$. However, even the generalised delayed feedback control is incapable of controlling any given system [7, 8]. It is not within the scope of this paper to review all methods of chaotic control, merely to highlight the differences of the proposed rate control of chaos method with, what to all intents and purposes, are the standard methods of control of chaos.

To improve the behaviour of the delayed control method, several methods to choose appropriate control parameters have been developed [9, 10] but none of these methods seem to function correctly unsupervised. The potential of unsupervised control of a chaotic system would imply that any stable system that becomes chaotic, due to changes in parameters, can be controlled to remain stable until adaptation of the parameters into non-chaotic domain is achieved. For example, a laser may become chaotic when additional optic elements are included, or when external interactions are present near the relaxation frequency [10]. If such a system would be controlled automatically as soon as a chaotic state was entered, the control would prevent the chaotic dynamics from affecting the performance too negatively, and the occurrence of the control would signal that a modulation of the parameters is required.

It is also conceivable that a controlled chaotic state, which is dynamically indistinguishable from a normal stable state when perturbed, is the natural state of a system. This may seem unnecessary, considering that normal stable states are sufficient for many systems, but it may be of benefit to some dynamic system where the existence of chaos (albeit controlled) allows more or better response to external input. For example, it may allow rapid switching between different stable states, or the selection of a specific state depending on the input. Alternatively, a switch from a stable state to a controlled chaotic state, and back to a stable state, may be used to overcome possible dynamically difficult transitions such as an energetically expensive move from one stable state to another. By temporarily switching to a controlled chaotic state the energy threshold may be vastly reduced, creating the opportunity to improve the performance of the system.

Previously, a method of chaotic control, that was derived from the concept of metabolic control, has been proposed [11]. The Rate Control of Chaos (RCC) method has demonstrated the principle of control of complex unstable dynamics, based on feedforward localised control. The results have shown that this would allow a nonlinear control of extracellular matrix formation to be stabilised, even though the intracellular mechanisms cannot observe directly the effects of the controller. This suggests potential mechanisms for control of more complex systems, and interactions between stable and unstable systems.

It is, therefore, of interest to explore the capabilities of this control method beyond the control of a single complex system, and investigate the behaviours of a controlled system when external perturbations are significant to the overall dynamics. In particular, the manner in which nonlinear controlled system may interact, and if this may result in a stable overall system. The interactions between RCC controlled systems, should address the dynamic behaviour of inherently stable connected systems, as well as inherently unstable systems, that also may become unstable due to diffuse connectivity.

To understand the mechanism of the RCC, and its relation to biochemical reaction dynamics, I will first discuss the RCC concept considered as a reaction kinetic mechanism, which will allow us to define the control function. Subsequently, I will discuss the RCC method as a dynamics limiting mechanism that will show its effect on the dynamic properties of a chaotic system, which will allow us to define the application of the control function to the 
chaotic system, resulting in a chaotically controlled system. This I will apply to the three dimensional Lorenz model, as well as higher order Lorenz models, to demonstrate its basic function of controlling a chaotic system, and analyse its effect on the controlled system. Furthermore, I will show how RCC performs in the presence of systemic noise. To emphasise the usefulness of the RCC method, I show that the control is also capable of controlling spatiotemporal chaos for two different spatiotemporal chaotic models, were the underlying dynamics can be stable periodic, as well as, controlled chaotic.

\section{RATE CONTROL OF CHAOS}

\section{A. RCC as a Reaction Kinetic Concept}

Biological systems, which are of tremendous complexity, are generally not chaotic unless pathological [12]. With the possible exception of neuronal systems, all biological systems are normally considered to be not chaotic. One may consider that these are therefore in stable states, and this appears to be often the case. In many situations, however, the complexity of interacting systems may prevent the simple determination of the parameter values that correspond to stable states. In such cases, it may be useful to develop appropriate parameter values by ensuring that possible chaotic states do not disrupt the system. By using a control mechanism that is only activated if the system diverges too fast from any given state (including transient states), the possibility of ending in a unstable state may be greatly reduced.

For this reason, one may consider a biological system to be a dynamically controlled system, based on localised control of individual chemical reactions that interact with, and perturb, other processes. If it is hypothesised that the essential biological control method ensures that the entire complex reaction system remains dynamically stable, the mechanism must have some properties that ensure this. The understanding of the enzymatic mediated reaction is based on the principles of Michaelis-Menten [13], and Briggs and Haldane [14]. More recently, the understanding of Metabolic Control Analysis [15] has aided the study of complex reaction networks, demonstrating the localised nature of the control of biochemical reaction kinetics.

Analysis of biochemical reaction kinetics can usually be resolved by a Quasi-Steady-State (QSS) assumption to reduce the complex dynamics [16]. The aim is to ensure that the system remains stable by considering the enzymesubstrate complex to be unvarying, due to the small amount of enzyme versus the large amount of substrate. The resulting expression is then greatly simplified, allowing good correspondence with experimental results. This approximation also permits the system to be consistently stable. For greatly reduced biochemical systems, and systems with relatively small amount of enzyme-substrate complexes, this works quite well. It is only in the detail of those systems, or when the relative quantities of substrate and enzyme are not very different, that the Quasi-Steady-State assumption cannot be maintained. However, it is important to the organism to maintain control of the biochemical system into some, seemingly, stable state to function reliably. A rate control assumption that stabilises the localised reactions dynamics could therefore be as effective as the QSS assumption.

The kinetic control of the rate equation in an enzyme mediated biochemical reaction is commonly expressed by the Michaelis-Menten model. The notion is the self-limiting rate of the substrate conversion by the enzyme complex into the product. The determination of the reaction velocity is based on the proportion of the available active enzyme, where the dissociation is assumed to be constant. This is comparable to the association of an acid, which also results in an asymptotic curve [13]. This idea can be applied to the change of the velocity of a dynamic variable. To do this, it is required to define the growth rate of such a variable as a proportion of its total ergodic capacity, to create a asymptotic limitation if the variable changes quickly. By borrowing the limitation function for a dynamic variable $x$, this proportional growth can be defined as the quotient $q_{x}$

$$
q_{x}=\frac{x}{x+\mu_{x}}
$$

where the constant $\mu_{x}$ represents the maximal capacity of the variable $x$. This function will asymptotically approach unity in one direction, and crossing zero to negative infinity when $-x$ equals $\mu_{x}$. Given that the variable $x$ is mapped onto the positive quadrants, we are only concerned with the approach to unity of $q_{x}$. To subsequently be able to employ this function as a rate limiting function, the response of the rate limiting function needs to be fast enough to overcome the inherent exponential growth of the unstable chaotic system. Therefore, the quotient $q_{x}$ is used as the argument in an exponential function that determines the appropriate increase or decrease in the rate of growth as

$$
\sigma(x)=f \exp \left\{\xi\left(q_{x}+\theta\right)\right\}
$$

where $f$ is a scalar, $\xi$ is the rate control parameter, and $\theta$ is an optional bias (normally zero). When the system is dynamically stable, or changes linearly, the relative change will cause the control function to approximate unity. 


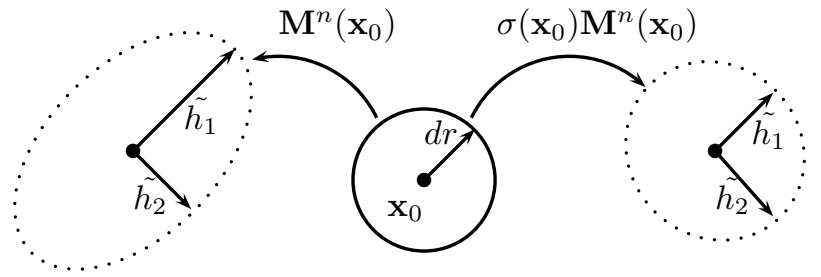

FIG. 1. Simple illustration of the effect of RCC on the evolution of a $2 \mathrm{D}$ sphere. At $\mathbf{x}_{0}$ (centre) after $n$ iterations of the map M, on the left, the exponential growth of the chaotic map (after [17]). On the right, the rate controlled map controlled into a stable state.

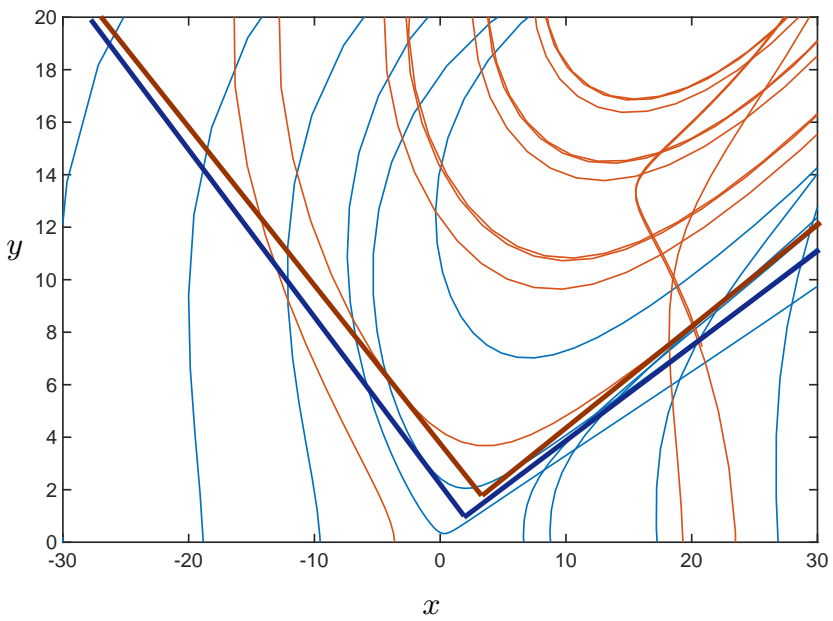

FIG. 2. Illustration of the local change in dynamic behaviour due to RCC in a dynamic flow. Several orbits of the chaotic Lorenz model (blue) and the RCC Lorenz model (red) are shown, with dark asymptotes illustrating the recognisable change in local dynamics.

Only when the system changes quickly will the control function deviate from this, allowing it to be applied as the rate control function to the variable $x$, limiting the growth rate. The proportional increase of the variable $x$ due to nonlinear change can be adjusted using the control function to limit the growth rate. The control function cannot be applied directly to the entire differential equation that defines $x$, as this may not be proportional to the growth rate, due to other terms in this equation. Equipped with the rate control function $\sigma$, it can now be applied to a dynamic chaotic system, by determining the appropriate rate limiting terms.

\section{B. RCC as a Dynamic Limiting Concept}

The RCC control function, described above, allows the control of the system to be an integral part of the chaotic system to be controlled, as a rate limiting function. It affects the dynamics of that system only marginally, only just enough to achieve the desired control. This may be considered as follows: the properties of stretching and folding of the chaotic attractor can be characterised by estimating the local Lyapunov exponents of the system. For an infinitesimal displacement from an initial condition $\mathbf{x}_{0}$ in the direction of a tangent vector $\mathbf{y}_{0}$, the Lyapunov exponent $h$ of the map $\mathbf{M}$ is defined by

$$
\begin{aligned}
h\left(\mathbf{x}_{0}, \mathbf{y}_{0}\right) & =\lim _{n \rightarrow \infty} \frac{1}{n} \ln \left|\mathbf{J}_{\mathbf{M}}^{n}\left(\mathbf{x}_{0}\right) \cdot \frac{\mathbf{y}_{0}}{\left|\mathbf{y}_{0}\right|}\right| \\
& =\lim _{n \rightarrow \infty} \frac{1}{n} \ln \left(\frac{\left|\mathbf{y}_{n}\right|}{\left|\mathbf{y}_{0}\right|}\right)
\end{aligned}
$$

where $\mathbf{J}_{\mathbf{M}}^{n}$ is the Jacobian matrix of the map at $n$ iterations. To provide a simple illustration of this, in figure $\mathbf{1}$ is shown the evolution of a two dimensional sphere after $n$ iterations of the map $\mathbf{M}$ with initial infinitesimal radius $r$. The resulting expansion in both directions is exponential and grows on average as $e^{n h_{1}}$, i.e. $\tilde{h}_{1}=e^{n h_{1}} d r$ [17]. The rate control method adjusts the rate of expansion using a non-linear control term (2) such that the system exponential growth is greatly reduced or eliminated. This is illustrated on the left side of figure $\mathbf{1}$, where is shown the chaotic iteration that grows exponentially in one direction. On the right side of the figure is illustrated the reduced controlled growth of the map due to the rate control function $\sigma\left(\mathbf{x}_{0}\right)$. The effect of the RCC on a flow is illustrated by the local change in the rate of the controlled Lorenz model (see also section IV), in comparison to the uncontrolled chaotic system. In figure $\mathbf{2}$ is shown some of the trajectories of the chaotic Lorenz model (blue) and the RCC controlled Lorenz model (red), with dark asymptotes demonstrating that controlled trajectories exist due to local changes in the rate of the system. The RCC control changes the rate of in the flow such that the exponential divergence is reduced, resulting in smoother trajectories locally.

The RCC method allows the control of a nonlinear system by controlling only those terms in an equation that contribute to the exponential growth of the variable. This need not be the nonlinear term in the system, but that 
term which permits the variable to increase exponentially the rate of that variable, e.g. another dependent variable that is nonlinear. The presence of terms that slow down the change in rate may also be effective, if some internal feedback loop exists within the entire set of equations.

Where the controlling term is nonlinear, the variables that form the growth term can be combined as the product of their quotients $\left(q_{x} \times q_{y}\right)$, which is then used to determine the final amount of control needed for that term using an exponent based on that product. If the variables that contribute to the control are in steady-state or stable oscillation, the control function will either be constant (near unity) or oscillate itself. For systems where the source of instability is readily separable, the RCC method will effectively control the system even if the system changes to different dynamic domains. The method employed by RCC does not target any specific stabilising orbit, but attempts to control the system when the chaotic wandering comes near a possible control domain. In effect, this means that the control is relatively neutral to the way it affects the system, and can remain active under normal conditions.

A RCC controlled system is stable in the sense of Lyupunov, where a controlled solution will remain in the controlled state or orbit. It is furthermore also asymptotically stable given that a controlled initial condition near a stabilised state or orbit will converge to the stable orbit or state (see section IV). The RCC controlled system can also be said to be exponentially stable, provided it is in the controlled state or orbit. Any perturbation away from the controlled orbit or state will converge back to the state at the controlled rate. However, due to the fact that the RCC does not target solutions, it requires the asymptotic convergence to a controlled state or orbit, it cannot be said at this point that it is exponentially stable under all conditions.

The determination of the control parameter values, and the terms to which to apply the control functions, remain somewhat non-obvious. This is primarily due to the fact that the controlled orbit is contained within the system, and cannot readily be identified a priori. Analytical methods may indicate the existence of an unstable periodic orbit within the strange attractor at a specific point, however, it is not guaranteed that the RCC method can control the system to that specific solution. When applying RCC to a specific system, an empirical approach by trial-and-error has been relatively easy and effective. It could be argued that some evolutionary search of potential control points, and parameter values, is in line with the biological interpretation of the concept.

\section{RCC OF LORENZ SYSTEMS}

The Lorenz system is an excellent example of a non-linear system with interesting dynamics, with different modes $[10,17]$. The bifurcation parameter $\rho$, in the Lorenz model, determines the shape and size of the attractor. This system is a classic example of chaos, and also interesting because of the different shapes the strange attractor can take. The system is known to be difficult to control with the OGY and delayed feedback methods [8]. Equations (8) to (10) show the three dimensional Lorenz system with rate control functions (5), (6) and (7). The control is included in the Lorenz system in equations (8), (9) and (10). These control functions are sufficient to control the Lorenz system through different bifurcations (by changing the parameter $\rho$ ), provided that the bias $\theta_{x y}=-0.05$. This is illustrated in figure 3 where the three dimensional Lorenz system is shown in the $x, z$-projection for three values of $\rho$ without control $(\xi=0)$. In figure 4 is shown the same three systems with RCC enabled. In each case, RCC stabilises the Lorenz system into a multi-orbit. As can be seen in figure 4, RCC is capable of controlling the system, within the attracting domain e.g. for the case (B) $\rho=110$, but can also stretch the system to include part of the phase-space where the control is more effective as for the cases $(\mathbf{A}) \rho=28$ and $(\mathbf{C})) \rho=210$. The Lorenz parameter values are $\gamma=10, \beta=2 \frac{2}{3}$, and the control parameters are shown in table I.

$$
\begin{aligned}
\sigma(y) & =f \exp \left\{\frac{\xi_{y}\left(y+\theta_{y}\right)}{(y+\mu)}\right\} \\
\sigma(x, z) & =f \exp \left\{\frac{\xi_{x z}\left(x z+\theta_{x z}\right)}{(x z+x+z+\mu)}\right\} \\
\sigma(x, y) & =f \exp \left\{\frac{\xi_{x y}\left(x y+\theta_{x y}\right)}{(x y+x+y+\mu)}\right\} \\
\frac{d x}{d t} & =\gamma(\sigma(y) y-x) \\
\frac{d y}{d t} & =\rho x-y+\sigma(x, z)(z-x) \\
\frac{d z}{d t} & =\sigma(x, y)(x y)-\beta z
\end{aligned}
$$




\begin{tabular}{|c|c|c|c|c|c|c|c|c|}
\hline$\rho$ & $f$ & $\xi_{y}$ & $\xi_{x z}$ & $\xi_{x y}$ & $\theta_{y}$ & $\theta_{x z}$ & $\theta_{x y}$ & $\mu$ \\
\hline 28 & -10 & -0.1 & -3 & -2 & 0 & 0 & -0.05 & 500 \\
110 & -3 & -1 & -3 & -3 & 0 & 0 & -0.05 & 500 \\
210 & -2 & -3 & -3 & -3 & 0 & 0 & -0.05 & 500 \\
\hline
\end{tabular}

TABLE I. Rate Control parameter values for different values of the Lorenz bifurcation parameter $\rho$.

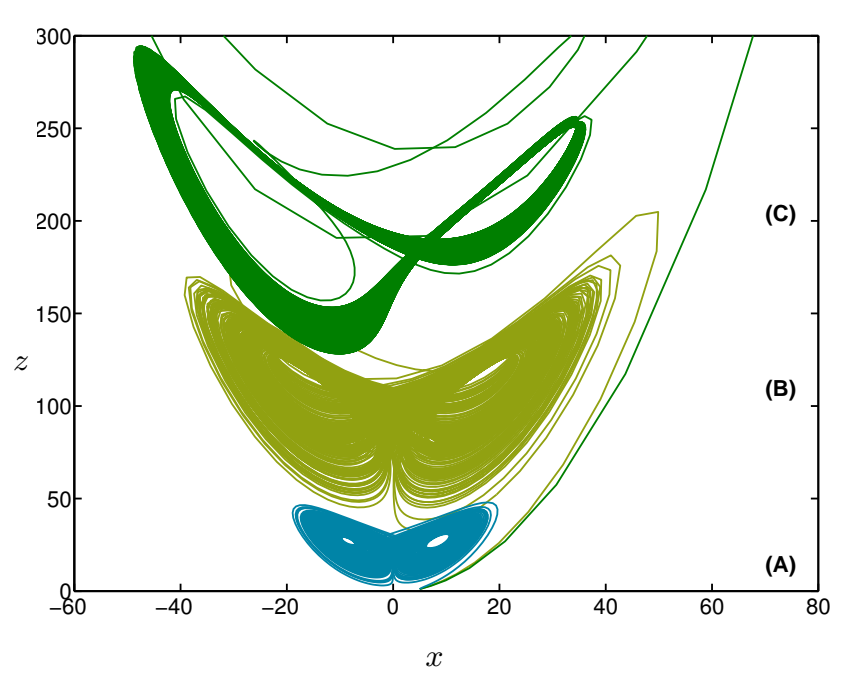

FIG. 3. Three forms of the chaotic three dimensional Lorenz model, for different values of the bifurcation parameter $\rho$; (A) $\rho=28$; (B) $\rho=110 ;$ (C) $\rho=210$.

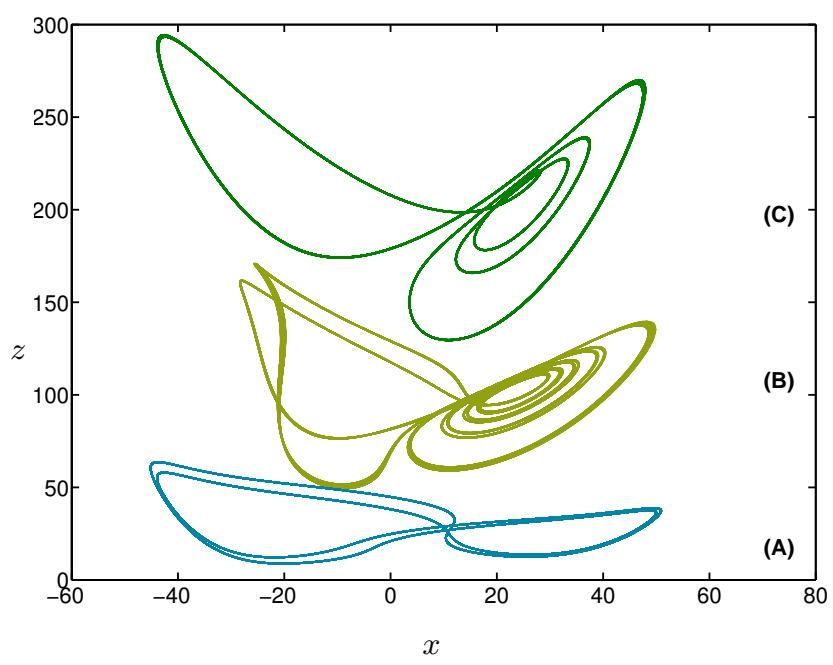

FIG. 4. Rate control of the three dimensional Lorenz model, for three values of the bifurcation parameter $\rho$; (A) $\rho=28 ;(\mathbf{B}) \rho=110 ;(\mathbf{C}) \rho=210$, resulting in three stable multi-orbits.

There exist also several hyper-chaotic forms of the Lorenz attractor $[18,19]$ which contain all the dynamic properties of the three dimensional system as a special case. For example, the addition of an extra feedback controller in the standard Lorenz model [18], creates a system that can demonstrate periodic orbits, chaos and hyper-chaos (with more than one positive Lyapunov exponent). This system is described by equations (12) to (15), with the control function (11). The inclusion of RCC can stabilise this system even more readily than the three dimensional Lorenz system, due to the presence of the feedback loop (see figure 5), which is why only one control function is sufficient. This hyper-chaotic Lorenz system clearly demonstrates how the rate control function slows down or speeds up depending on the dynamics of the system (figure $\mathbf{6}$ and figure $\mathbf{7}$ ). The rate control function will be larger than unity when the rate is increased and smaller when it is slowed down. Also note in figure 6 that the system will take some time to converge to the stable oscillation after control has been enabled at time $t=50$. The convergence rate is not strictly related to the control function, but appears to be determined by the chaotic drift, and may vary depending on the state of the system when control is enabled. Parameter values for the chaotic system are $\chi=10, \beta=8 / 3, \gamma=0.1, \rho=28$, and the bifurcation parameter $k=3$ is in the hyper-chaotic domain. RCC parameters are $\xi=2, \theta_{y z}=0, \mu_{y z}=100$ and $f=1$.

The RCC method does not simply remove all aspects of chaos from the controlled system. In figure $\mathbf{8}$ can be seen the rate control function versus the local Lyapunov exponent estimate [20] for a single controlled multi-orbit. Where the RCC function does not greatly affect the local dynamics, the Lorenz system can still be weakly chaotic as shown by the small positive Lyapunov exponent estimates. The control affects the local Lyapunov exponent by adjusting the local chaotic growth, except where the exponential growth rate is faster than the RCC. This, however, allows different orbits to be controlled for different parameters of the control function (primarily $\xi$ ), where the different RCC control functions may still maintain a stable orbit. Furthermore, this property confirms that the RCC method is a true method of control of chaos, where small adjustments are made to the system to stabilise it, without major changes to the underlying chaotic system. Additionally, it provides the means for asymptotic stability, due to the fact that it changes the rate only if divergence is under way. This can be further explored by perturbing the system, which will be shown subsequently. 


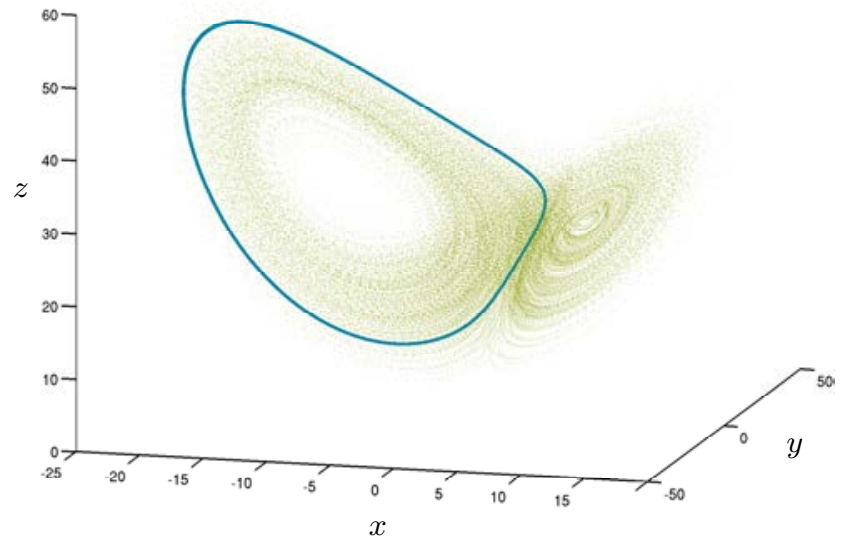

FIG. 5. 3D-plot of the four dimensional Lorenz model, showing chaos and RCC.

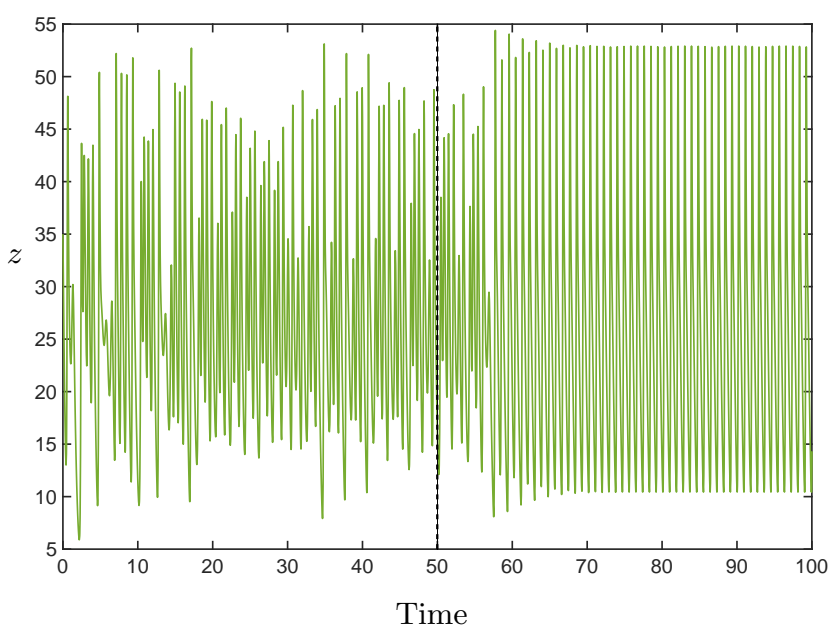

FIG. 6. Evolution plot of the $z$ variable of the four dimensional Lorenz model showing chaos with RCC enabled at $t=50$.

$$
\begin{aligned}
\sigma(y, z) & =f \exp \left\{\frac{\xi\left(y z+\theta_{y z}\right)}{\left(y z+y+z+\mu_{y z}\right)}\right\} \\
\frac{d x}{d t} & =\chi(y-x) \\
\frac{d y}{d t} & =\rho x+y+(z-x)-w \\
\frac{d z}{d t} & =(x y)-\beta z \\
\frac{d w}{d t} & =\sigma(y, z) \gamma(x y)+k
\end{aligned}
$$

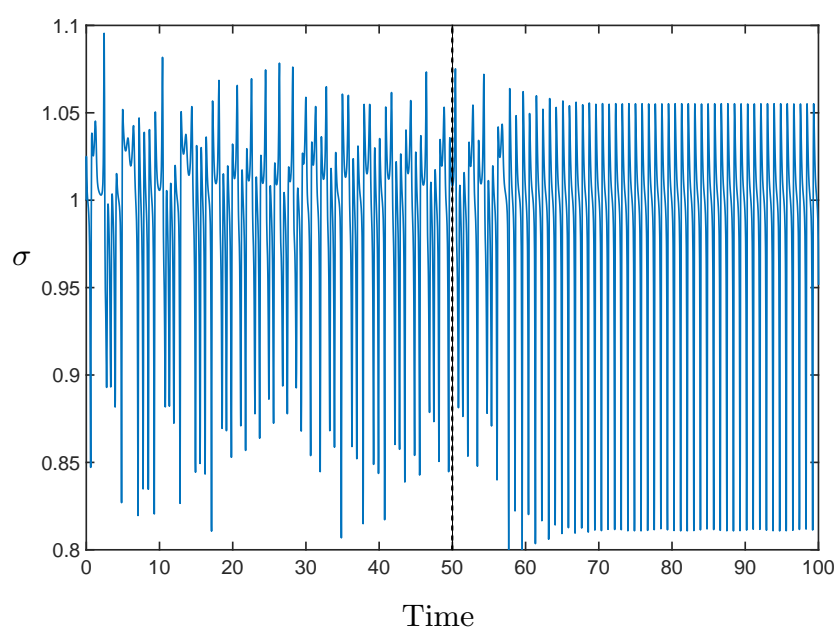

FIG. 7. Evolution of the RCC function $\sigma$ of the four dimensional Lorenz system with control enabled at $t=50$, where the system is quickly controlled and stable oscillating.

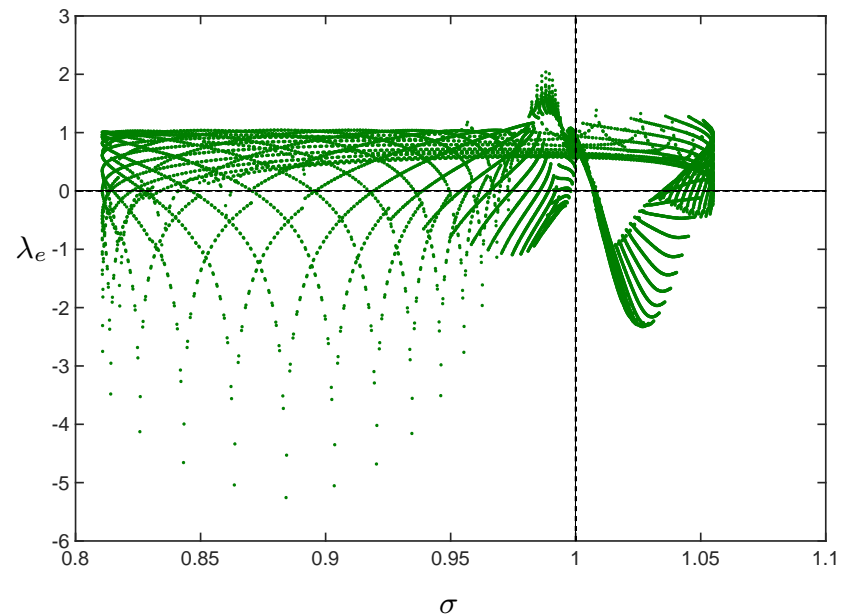

FIG. 8. Plot of the local Lyapunov estimate $\lambda_{e}$ of the controlled four dimensional Lorenz system, versus the RCC function $\sigma$, showing that the controlled system is still weakly chaotic $\left(\lambda_{e}>0\right)$. 


\section{NOISE ROBUSTNESS}

The RCC method of control is quite robust against systemic noise that may be present in the chaotic system. This may be understood by considering that the control function (11) changes due to the local change in the rate of the control variable. If this change is due to noise, rather than due to nonlinearity, this will simply cause the control function to expect the change to be due to exponential divergence. The control will then adjust the rate to compensate for this. This, of course, means that the system noise will also be present in the control function, but significantly reduced. If the noise becomes too large for the control function to be able to stabilise the system, it will simply fail to achieve stability. To demonstrate this property, the four dimensional Lorenz model [18] has been modified to include Gaussian noise with variable distributions. In this example, equation(12) has been modified to add normalised Gaussian noise $\epsilon$ to the $x$ variable.

$$
\frac{d x}{d t}=\chi(y-x)+\epsilon
$$

In figure 9 is shown the $x$ versus $y$ plot of this system with 1,2 and 4 standard deviations (S.D.) of normalised Gaussian noise added. Here is shown, superimposed, the blue dots of the RCC controlled orbit with 4 S.D., green dots with 2 S.D., and the red dots 1S.D. noise of variable $x$ versus $y$. Similarly, in figure 10 is shown the same stable orbit of the same different amounts of noise, but the $x$ variable is plotted against the control function $\sigma$. It shows how the added noise to the chaotic system causes the control function to adjust the amount of control.

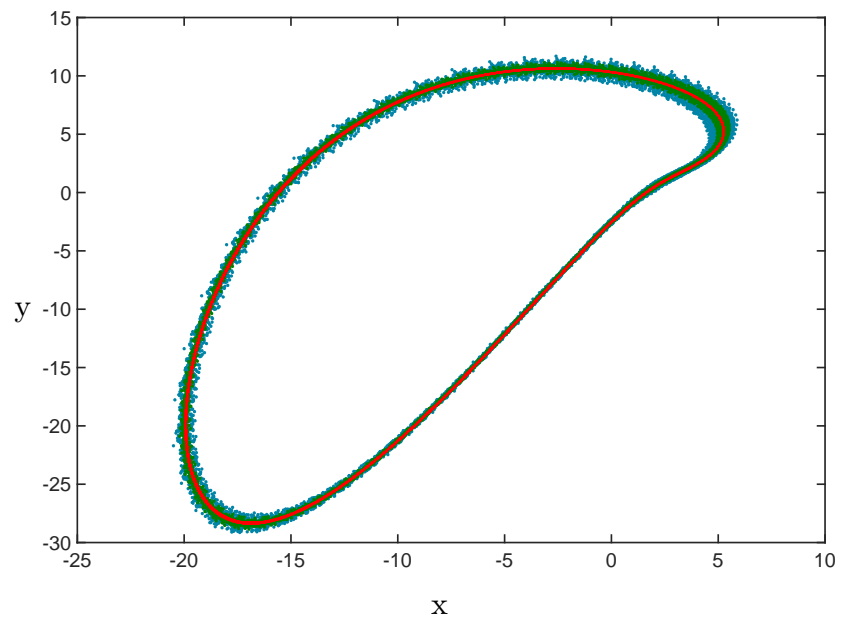

FIG. 9. Plot of the noisy RCC controlled four dimensional Lorenz model showing variables $x$ versus $y$. Red dots, green dots, and blue dots indicate the amount of Gaussian noise, 1, 2 and 4 standard deviations from the normalised distribution respectively.

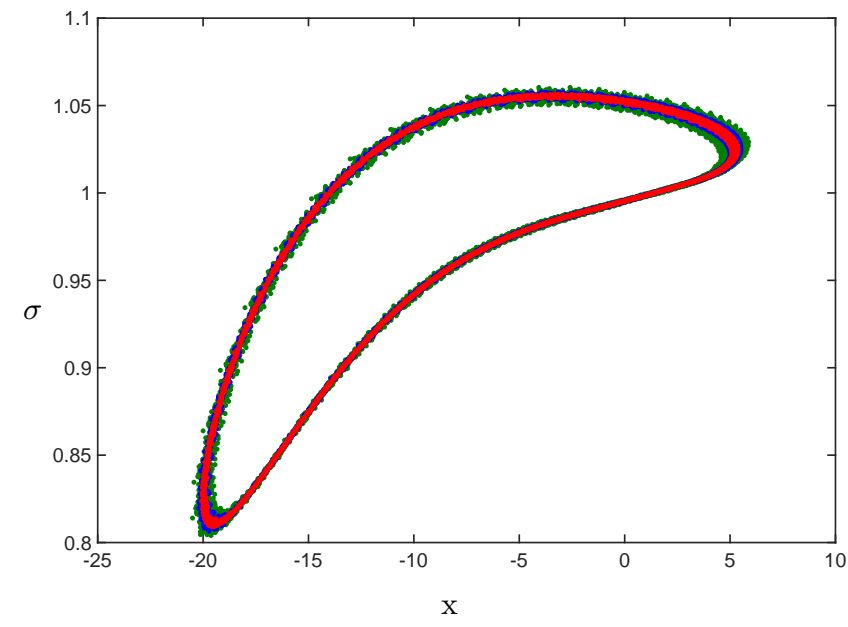

FIG. 10. Plot of the $x$ variable with added noise versus the control function $\sigma$. Red dots, green dots, and blue dots indicate the amount of Gaussian noise, 1, 2 and 4 standard deviations from the normalised distribution respectively.

To clarify the effect noise has on the RCC controlled system, a detailed figure of the top right hand corner of the orbits in figure 9 is shown in figure 11. Here, the RCC orbit is shown in black, with the 4 S.D. normalised Gaussian noise in blue superimposed. As can be seen, the exact orbit the system follows is different, depending on the amount of perturbation, but remains in the same spatial domain as the unperturbed orbit. The noise causes the orbit to be drift around the target orbit, but the RCC will adjust this at the same rate as the exponential divergence due to chaos. Even for moderate amounts of noise, for this type of system, it will be somewhat successful, if the system needs to remain within a specified window. In figure $\mathbf{1 2}$ is plotted a short segment of the control function for the noise perturbed system $\sigma$ versus the noise $\epsilon$ applied to the system. Instead of oscillating around unity, as can be seen in figure $\mathbf{7}$, the control function will fluctuate to accommodate the noise.

This demonstrates that the RCC results in a dynamically stable system, even when perturbed with Gaussian noise. The stability introduced by RCC appears to be at least asymptotically stable, and may be exponentially stable for a neighbourhood around the system. The size of the neighbourhood will depend on the nature of the system, and the ability of RCC to reach the domain of control. Whilst the RCC is attempting to stabilise the system, the perturbation may prevent the system from stabilising if a large neighbourhood of noise or perturbation exists. 


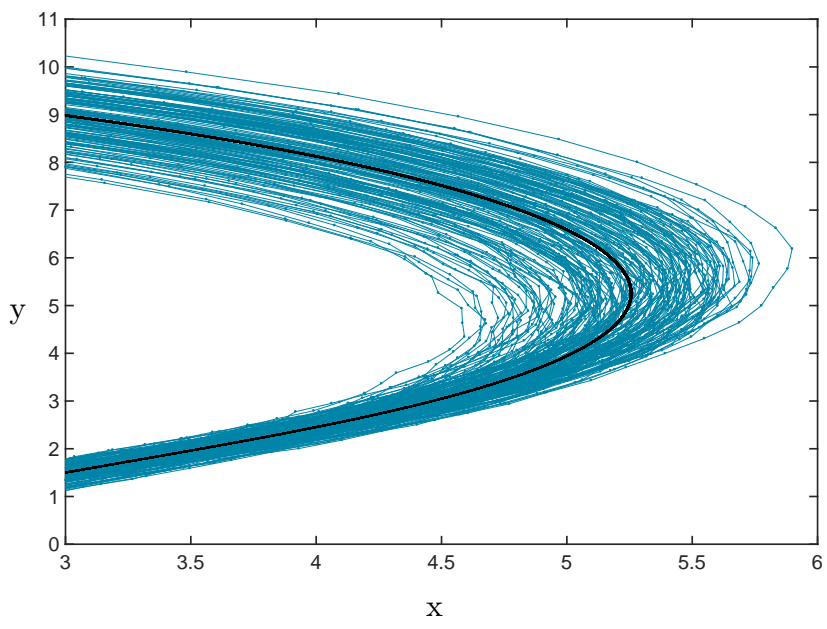

FIG. 11. Detail of the noisy RCC controlled four dimensional Lorenz model, showing how the noise causes the orbit to vary, yet the control maintains the local dynamic behaviour of the system. The black line is the noiseless controlled orbit, the blue lines are the 4 S.D. perturbed orbits.

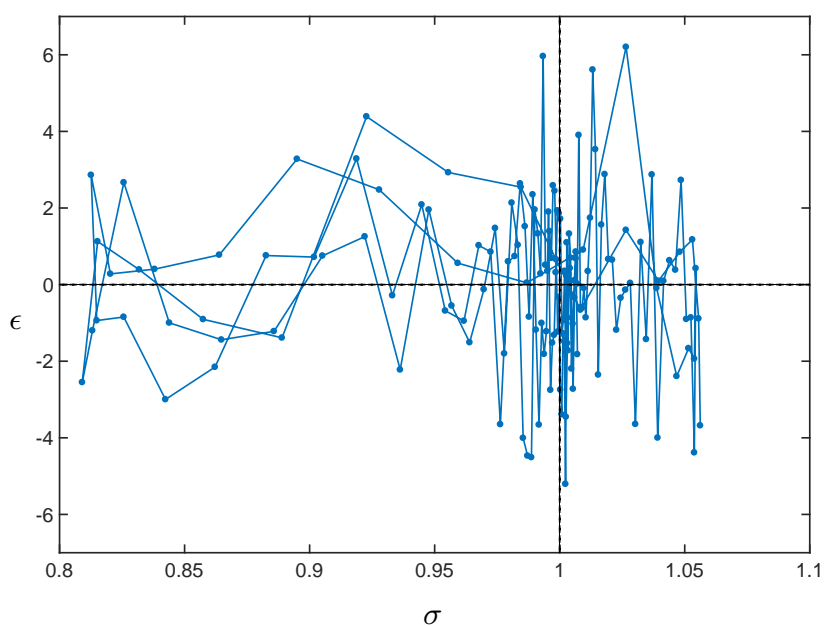

FIG. 12. Short segment of the control function $\sigma$ versus the noise signal. The control should cycle periodically around unity, but the noise causes it to wander (cf. figure 7).

\section{RCC OF SPATIOTEMPORAL CHAOS}

Spatiotemporal chaos is a specific form of chaotic complex behaviour that emerges from interactions between elements in a spatial organisation. The underlying dynamics of each element can be stable, periodic or even chaotic, depending on the choice of the system. It has been shown that simple oscillating models of Turing patterns in reaction-diffusion systems can readily become unstable and show emerging chaotic dynamics in a spatial pattern [21]. A generalised OGY method of control has been employed to control spatiotemporal systems [22] and further extended to control Mixed-Mode Spatiotemporal chaos [23]. These methods require continuous observation of the system to commit localised perturbations system at predetermined times and locations.

The RCC method can be applied to these systems, which removes the need to supervise the dynamics of the chosen elements in the spatiotemporal lattice. As is shown below, RCC controls the dynamics of the chaotic system, but also ensures that the system maintains stable through bifurcations that would cause the uncontrolled system to become hyper-chaotic. This feature of the rate control method will allow a system to be controlled relatively independent of the underlying dynamic state of the system. These underlying dynamics could be simple steady-state processes or even chaos or hyper-chaos. Without a methodology such as RCC, these systems will never be easily controllable. It may be possible to tune a feedback or Proportional-Integral-Derivative (PID) controller for specific instances, but it is known that these are never practically robust [24]. Robustness and stability over changing dynamics by using RCC can be shown in spatiotemporal chaos in two different reaction-diffusion systems, as follows.

\section{A. Gray-Scott chemical model}

In the Gray-Scott autocatalytic model (19) and (20), mixed-mode spatiotemporal chaos emerges from the interaction between Turing and Hopf bifurcations [23, 25]. Using RCC this system can be readily controlled without the need to supervise the system. The system is modelled using finite differences for a $1 \mathrm{D}$ case with no-flux boundary conditions, both with RCC and for uncontrolled chaos. 


$$
\begin{aligned}
\sigma\left(\alpha_{n}\right) & =\exp \left\{\xi \frac{\alpha_{n}}{\alpha_{n}+\mu_{n}}\right\} \\
\sigma\left(\beta_{n}\right) & =\exp \left\{\xi \frac{\beta_{n}}{\beta_{n}+\mu_{n}}\right\} \\
\frac{\partial \alpha_{n}}{\partial t} & =\delta \frac{\partial^{2}}{\partial x^{2}} \alpha_{n}+\frac{\sigma\left(\alpha_{n}\right)\left(1-\alpha_{n}\right)}{T_{\text {res }}}-\alpha_{n} \beta_{n}^{2} \\
\frac{\partial \beta_{n}}{\partial t} & =\frac{\partial^{2}}{\partial x^{2}} \beta_{n}+\frac{\sigma\left(\beta_{n}\right)\left(\beta_{0}-\beta_{n}\right)}{T_{\text {res }}}+\alpha_{n} \beta_{n}^{2}-k \beta_{n}
\end{aligned}
$$

where $\delta=\frac{D_{\alpha}}{D_{\beta}}=4.6$ is the ratio of the diffusion coefficients of the reactant and the autocatalyst, $\beta_{0}=1 / 15, k=1 / 40$ for $n \in\{1, \ldots, 10\}$. The systems are coupled in 1D by

$$
\begin{aligned}
\frac{\partial^{2}}{\partial x^{2}} \alpha_{n} & =G_{n}\left(\alpha_{n+1}-\alpha_{n}\right)+\left(\alpha_{n-1}-\alpha_{n}\right) \\
\frac{\partial^{2}}{\partial x^{2}} \beta_{n} & =G_{n}\left(\beta_{n+1}-\beta_{n}\right)+\left(\beta_{n-1}-\beta_{n}\right)
\end{aligned}
$$

where $G_{n}=0.005$, and optional boundaries conditions. The RCC functions (17) and (18) control the rates of the two reactants $\alpha$ and $\beta$. The parameter $T_{r e s}$ is a bifurcation parameter, and a Turing pattern emerges over the range $214.8<T_{r e s}<242$. At lower values, the stable oscillating Turing pattern becomes unstable [23], here $T_{\text {res }}=214.5$ has been chosen, well within the chaotic domain. The addition of RCC for the stable oscillating system has little effect on the oscillations, but once control is enabled for the chaotic case, the system rapidly stabilises. This is illustrated in figure 13 where the spatiotemporal chaos of the uncontrolled system is shown, with the RCC control enabled at simulated time $t=800$. The occasional chaotic disintegration of the oscillating pattern in the chaotic case, is stabilised by RCC into a lower amplitude oscillation with $\xi=0.2, \mu_{n}=1$. The RCC control applied locally at each oscillator, causes the system to readily adapt to the perturbing diffusion from other oscillators which, for the chaotic case, would destabilise the entire system. The local control of RCC does, therefore, not depend on a supervisor, and can also adjust for external perturbations where applicable, which makes it relatively noise insensitive.

The effect of changes in the control parameter $\xi$, can be seen in figure 14, where the increase in the control parameter $\xi=0.4$ shows a stable oscillation but at lower amplitude than in figure 13. By determining the Lyapunov estimate for the variables $\alpha_{n}$ and $\beta_{n}$ for the 1D case, the control of RCC can be visualised. In figure $\mathbf{1 5}$ can be seen the estimate for $\beta_{n}$, based on 20 Lyapunov steps [20], with large positive variations for the uncontrolled case, with small positive values for the controlled case. Repeating this for $\alpha_{n}$ in figure $\mathbf{1 6}$ the estimates show large negative variations for the uncontrolled case, and small oscillating values for the controlled case. This can be more clearly seen in figure 17, where the Lyapunov estimates are shown for a single system at position 5, i.e. the estimate for the variables $\alpha_{5}$ and $\beta_{5}$. This shows that the estimate is positive for the variable $\beta_{5}$ and negative $\alpha_{5}$ throughout, but the control reduces the local estimate to a smaller oscillatory value.

In spatiotemporal chaos, the connectivity strength is important with regards to the resulting dynamics. To demonstrate the robustness of the RCC to the system, the connectivity strength $G_{n}$ between Gray-Scott oscillators has been varied from $G_{n}=0.0005$, to $G_{n}=0.0025, G_{n}=0.0075$ and $G_{n}=0.01$. The resulting dynamics can be seen in figures 18, 19, 20, and 21, respectively. Given the constant value for the bifurcation parameter $T_{\text {res }}=214.5$, the connectivity shows oscillations for the lower range of the connectivity, until the chaotic intermittency shown in figure $\mathbf{1 3}$ and figure 20, before returning to high frequency oscillations in figure $\mathbf{2 1}$. Note that RCC is capable of controlling all the different scenarios due to the variability in the connectivity, but with different stabilised oscillatory solutions.

\section{B. Belousov-Zhabotinsky reaction}

When considering spatiotemporal chaos, the well known chaotic Belousov-Zhabotinsky (BZ) reaction can also be used to create more complex chaotic spatiotemporal dynamic behaviour, steady-state, oscillations, chaos and even hyper-chaos. It has been shown that the BZ reaction system can be controlled using a proportional feedback algorithm [26], but here it is shown for the more complex case of BZ with spatiotemporal dynamics. Using the Györgyi and Field model $[27,28]$ for a $1 \mathrm{D}$ case with no-flux boundary conditions and finite differences [26], it is shown that even when the BZ system goes through spatiotemporal bifurcations, the RCC method maintains control in all cases, including 


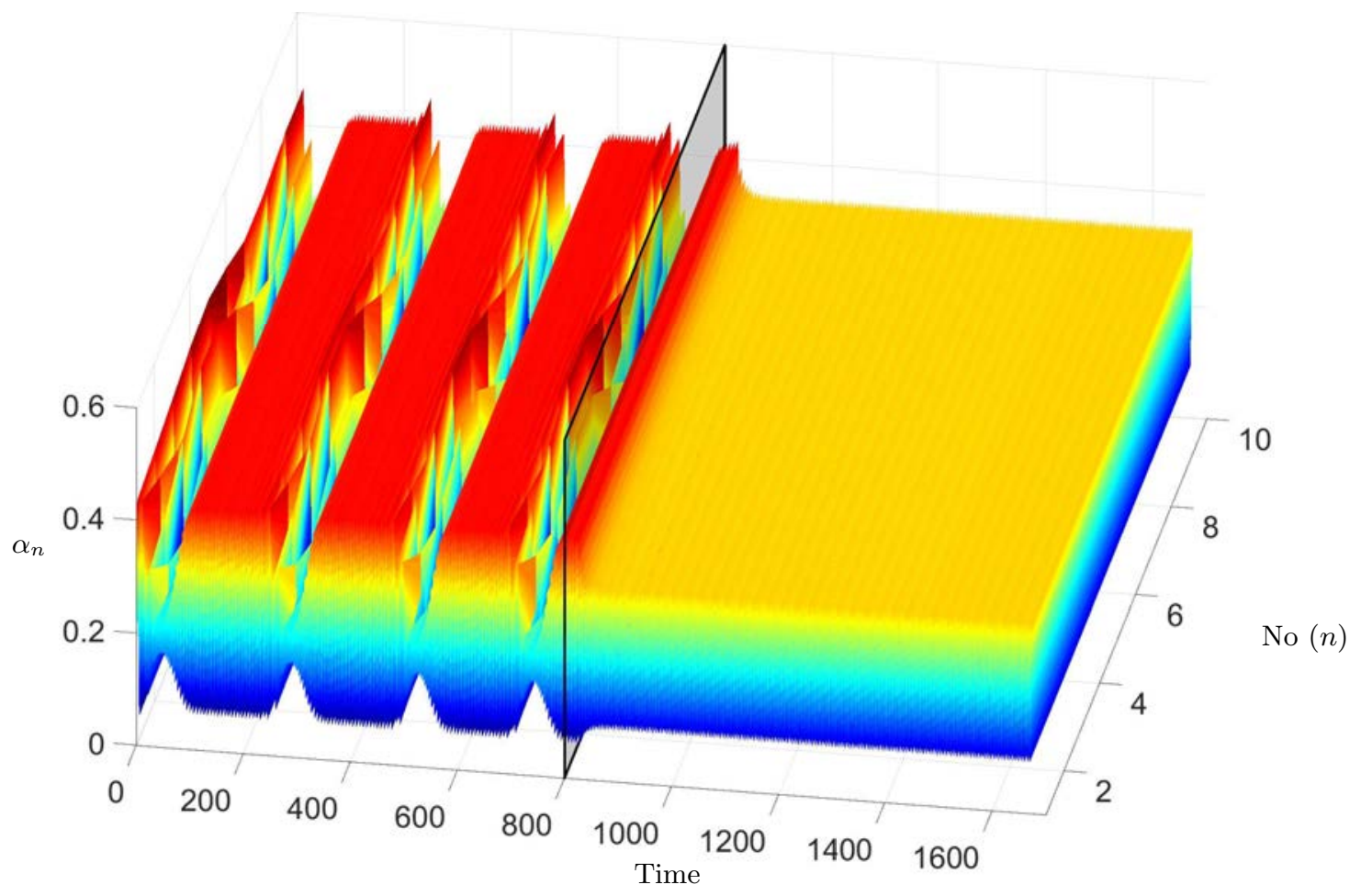

FIG. 13. RCC of the spatio-temporal 1D Gray-Scott system $(n=10)$. Initially, the chaotic system without control shows semi-stable patterns collapsing into chaotic bursts. RCC is here enabled at $t=800$, which results in stable oscillations.

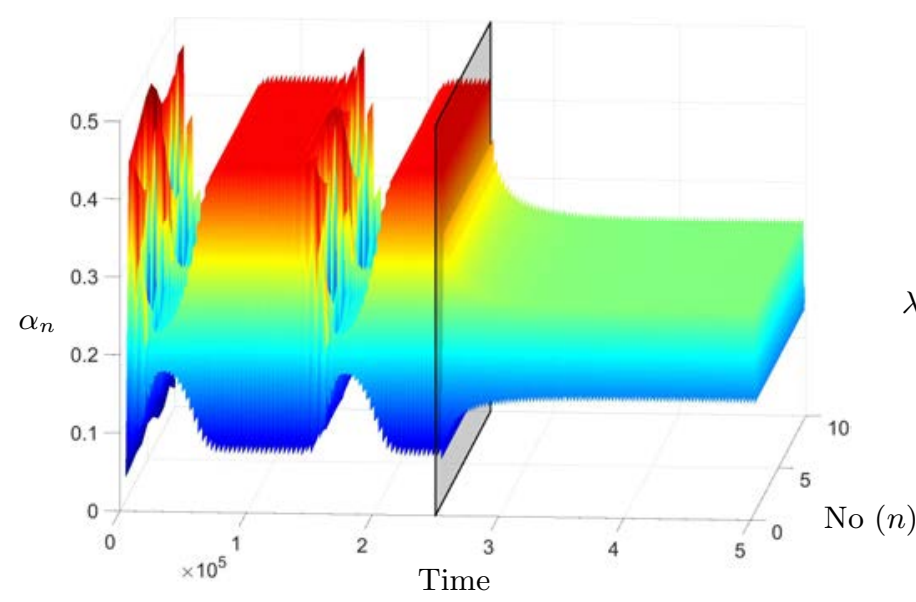

FIG. 14. RCC of the 1D Gray-Scott system, with $\xi=0.4$ enabled halfway through at $t=250000$ simulated time, showing stable smaller amplitude oscillations.

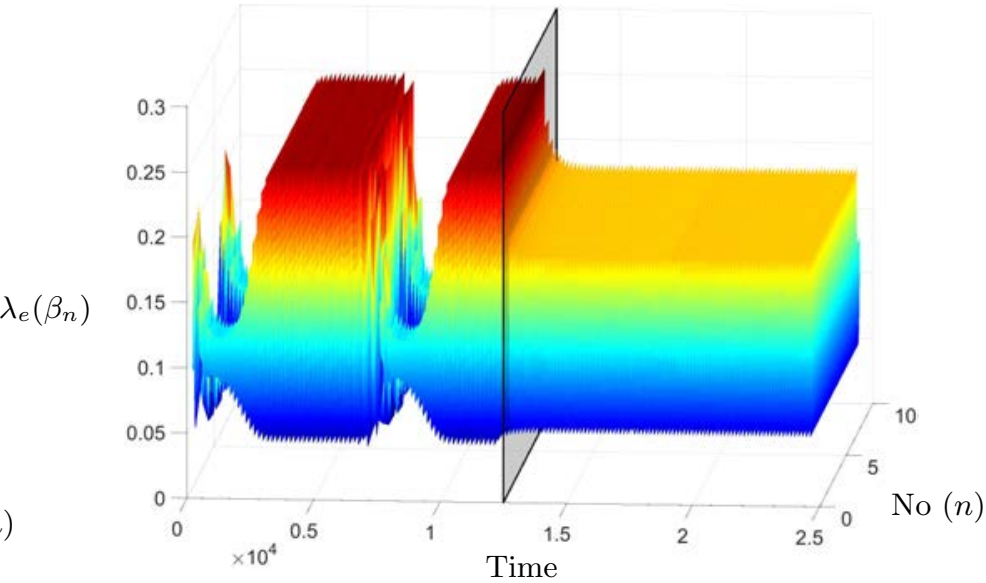

FIG. 15. Lyapunov estimates $\lambda_{e}$ for the variables $\beta_{n}$, showing higher positive estimates before control is enabled $(t=12500$, for 20 Lyapunov steps), followed by lower positive values during RCC.

hyper-chaos. These simulations are shown in figure $\mathbf{2 2}$ for the chaotic case, and figure $\mathbf{2 4}$ for the RCC controlled cases, where the capital letters refer to the state of the BZ reactions as determined by the bifurcation parameter $k_{f}$ [28] (see table II). The change in the bifurcation parameter $k_{f}$ causes the BZ reaction to be stable oscillating (states $\mathrm{A}$ and $\mathrm{B})$ or chaotic (C-F). Due to spatiotemporal diffusion of the reactants, the BZ $1 \mathrm{D}$ model becomes even more unstable in state $\mathrm{D}$ than the low dimensional chaos in case $\mathrm{C}$. In figure 22 at about $t \approx 140$ (D), the chaotic BZ reactions interact into a hyper-chaotic pattern. This pattern remains when the parameter $k_{f}$ is changed further. Once $\mathrm{RCC}$ is applied to each BZ reaction, the entire system is controlled and remains controlled through the parameter 


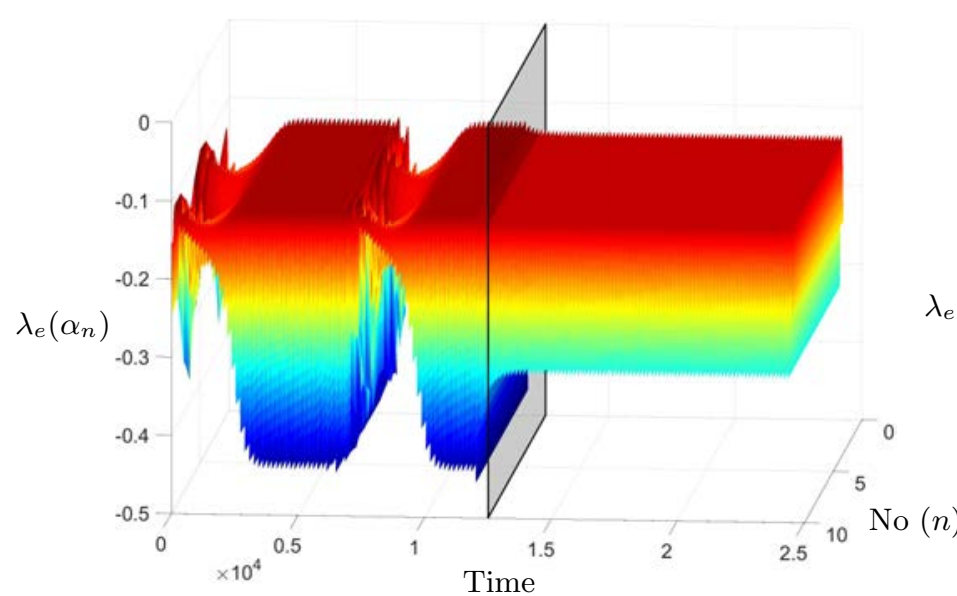

FIG. 16. Lyapunov estimates $\lambda_{e}$ for the variables $\alpha_{n}$, showing negative estimates throughout, but with smaller variability during RCC (enabled at $t=12500$, for 20 Lyapunov steps).

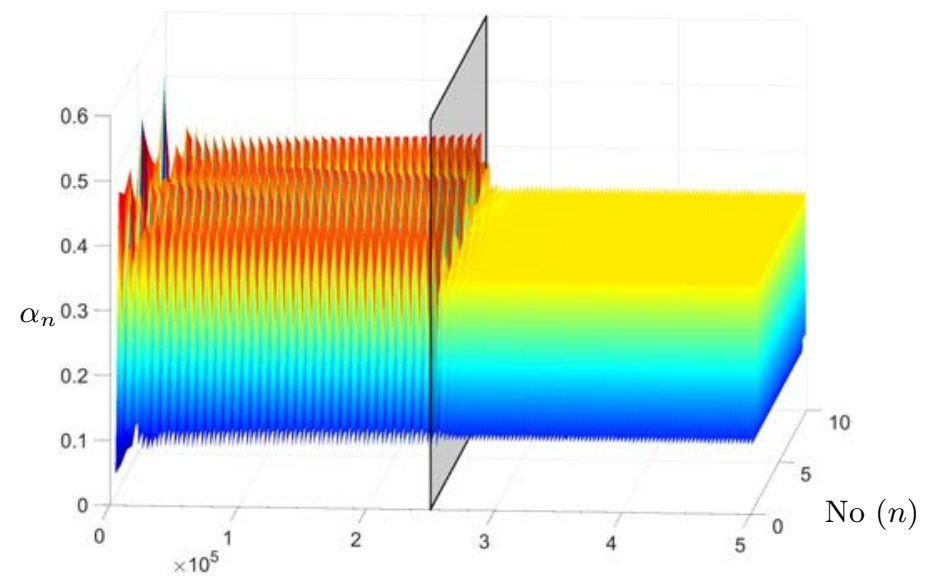

Time

FIG. 18. Effect of connectivity term $G_{n}=0.0005$, showing higher frequency oscillations before control is enabled, and a lower amplitude oscillation with RCC enabled $(t=250000)$.

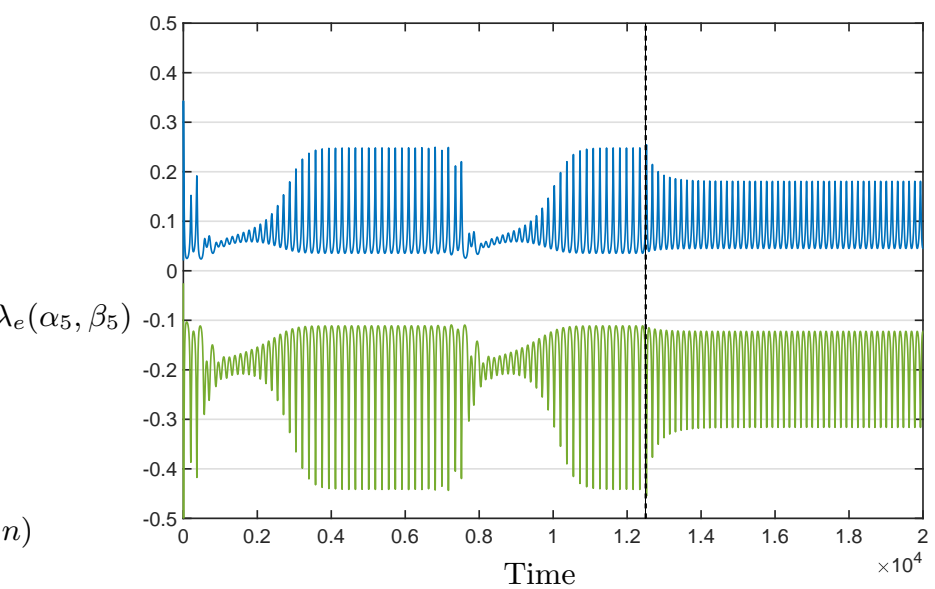

FIG. 17. Lyapunov estimates of the variables $\alpha_{5}$ (green, bottom), and $\beta_{5}$ (blue, top), showing larger variability during the uncontrolled phase, followed by smaller amplitudes oscillations of the estimates during RCC $(t=12500$, for 20 Lyapunov steps).

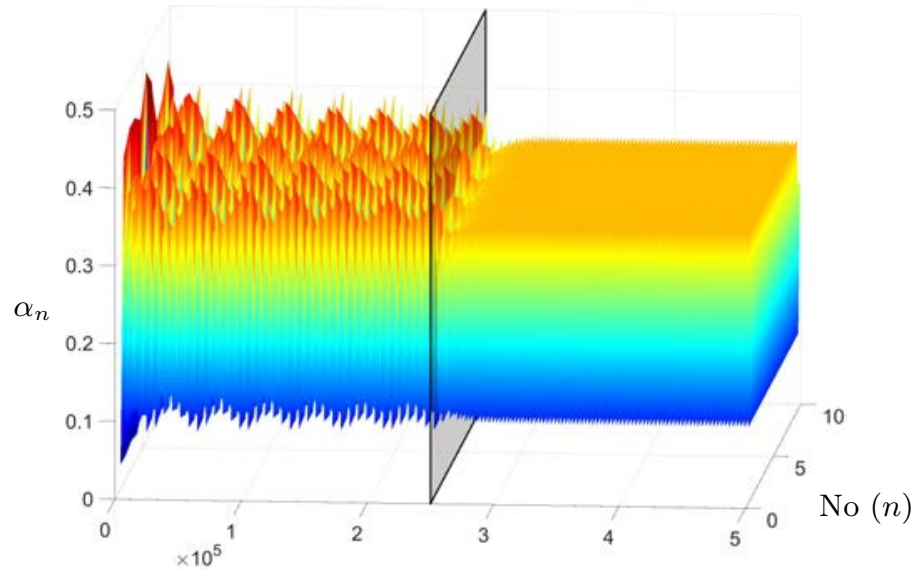

Time

FIG. 19. Effect of lower connectivity term $G_{n}=0.0025$, showing out of phase phase-locked oscillations before control is enabled, and an in-phase oscillation with RCC enabled $(t=250000)$.

changes, and quickly stabilises into oscillation patterns. For different RCC parameters (shown is $\xi=-0.5, \mu=3$ ), different oscillation patterns can be achieved. Although RCC can modify the underlying stable oscillation (as is the case with the Lorenz system), the qualitative dynamic behaviour of the system has not changed and allows the RCC to be enabled even when the system is already stable (see also [11]). The equations (24) to (27) describe the controlled Belousov-Zhabotinsky system, and (23) is the appropriate RCC function.

The corresponding Lyapunov estimates for the uncontrolled and the RCC controlled system can be seen in figure $\mathbf{2 3}$ and figure 25, which shows larger variability in the estimates for the uncontrolled case, but more reduced variability for the RCC case. The maximal Lyapunov estimates for the uncontrolled case is $\lambda_{\max }=0.6181$, and for RCC $\lambda_{\max }=0.6035$ througout the entire simulation. This shows that the control affects the stability of the system but does not greatly change the global dynamics, but only the local dynamics of each individual oscillator. 


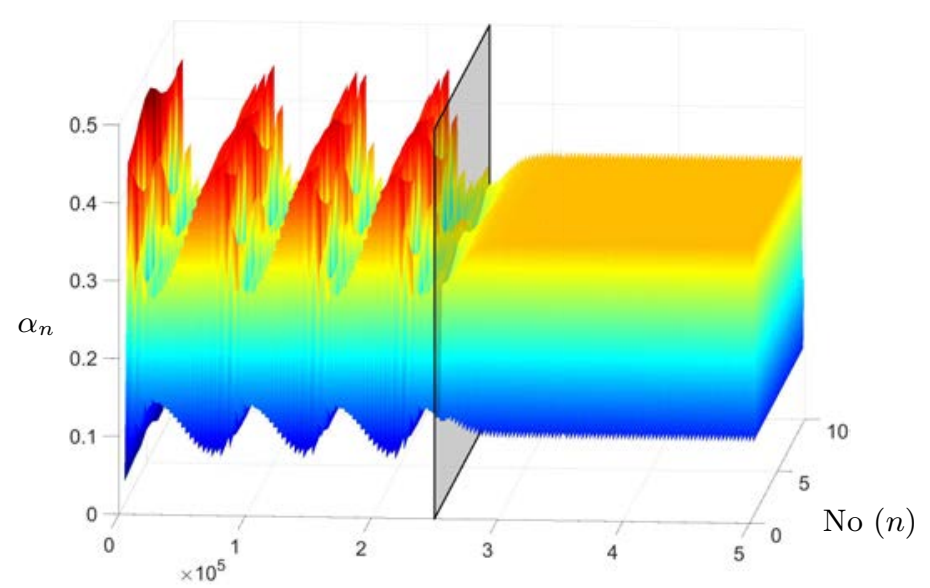

Time

FIG. 20. Effect of connectivity term $G_{n}=0.0075$, showing chaotic intermittency before control is enabled, and a stable oscillation with RCC enabled $(t=250000)$.

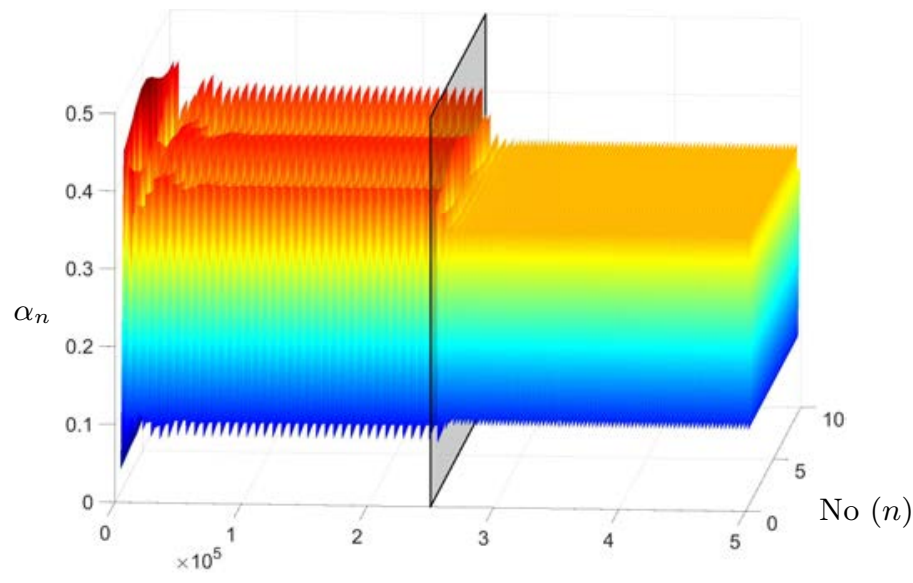

Time

FIG. 21. Effect of connectivity term $G_{n}=0.01$, showing high frequency oscillations before control is enabled, and similar oscillations with RCC enabled as in figure $19(t=$ 250000).

\begin{tabular}{|c|c|l|l|}
\hline State & $k_{f}$ & Uncontrolled dynamics & RCC dynamics \\
\hline A & $4.0 \times 10^{-4}$ & 2 orbit & 2 orbit \\
B & $3.8 \times 10^{-4}$ & 3 orbit & 1 orbit \\
C & $3.6 \times 10^{-4}$ & Chaos & 1 orbit \\
D & $3.4 \times 10^{-4}$ & Chaos \& Hyper-chaos & 3 orbit \\
E & $3.2 \times 10^{-4}$ & Hyper-chaos & 2 orbit \\
F & $3.0 \times 10^{-4}$ & Hyper-chaos & 1 orbit \\
\hline
\end{tabular}

TABLE II. States of the Finite-Difference-Belousov-Zhabotinsky reaction model of the uncontrolled system (third column) and the RCC controlled system (last column) for different values of the bifurcation parameter $k_{f}$.

$$
\begin{aligned}
\sigma_{n}\left(x_{n}\right) & =\exp \left\{\frac{\xi x_{n}}{x_{n}+\mu}\right\} \\
y_{n} & =\left(\frac{\alpha k_{6} Z_{0} V_{0} z_{1} v_{1}}{k_{1} H X_{0} x_{1}+k_{2} A H^{2}+k_{f}}\right) / Y_{0} \\
\frac{\partial x_{n}}{\partial t} & =T_{0}\left(-k_{1} H Y_{0} x_{1} y_{1}+k_{2} A H^{2}\left(Y_{0} / X_{0}\right) y_{n}\right. \\
& -2 k_{3} X_{0} x_{1}^{2}+0.5 k_{4} A^{0.5} H^{1.5} X_{0}^{-0.5}\left(C-Z_{0} z_{n}\right) x_{n}^{0.5} \sigma_{n}\left(x_{n}\right) \\
& \left.-0.5 k_{5} Z_{0} x_{n} z_{n}-k_{f} x_{n}\right) \\
\frac{\partial z_{n}}{\partial t} & =T_{0}\left(k_{4} A^{0.5} H^{1.5} X_{0}^{0.5}\left(C / Z_{0}-z_{n}\right) x_{n}^{0.5} \sigma_{n}\left(x_{n}\right)\right. \\
& \left.-k_{5} X_{0} x_{n} z_{n}-a k_{6} V_{0} z_{n} v_{n}-b k_{7} M z_{n}-k_{f} z_{n}\right) \\
\frac{\partial v_{n}}{\partial t} & =T_{0}\left(2 k_{1} H X_{0}\left(Y_{0} / V_{0}\right) x_{n} y_{n}+k_{2} A H^{2}\left(Y_{0} / V_{0}\right) y_{n}\right. \\
& \left.+k_{3}\left(X_{0}^{2} / V_{0}\right) x_{n}^{2} \sigma_{n}\left(x_{n}\right)-a k_{6} Z_{0} z_{n} v_{n}-k_{f} v_{n}\right)
\end{aligned}
$$

where $\sigma_{n}\left(x_{n}\right)$ is the RCC function and the subscript $n \in\{1, \ldots, 10\}$ indicates the index of each BZ reaction in 1D space (for the BZ chaotic system parameter values, see [28]). 


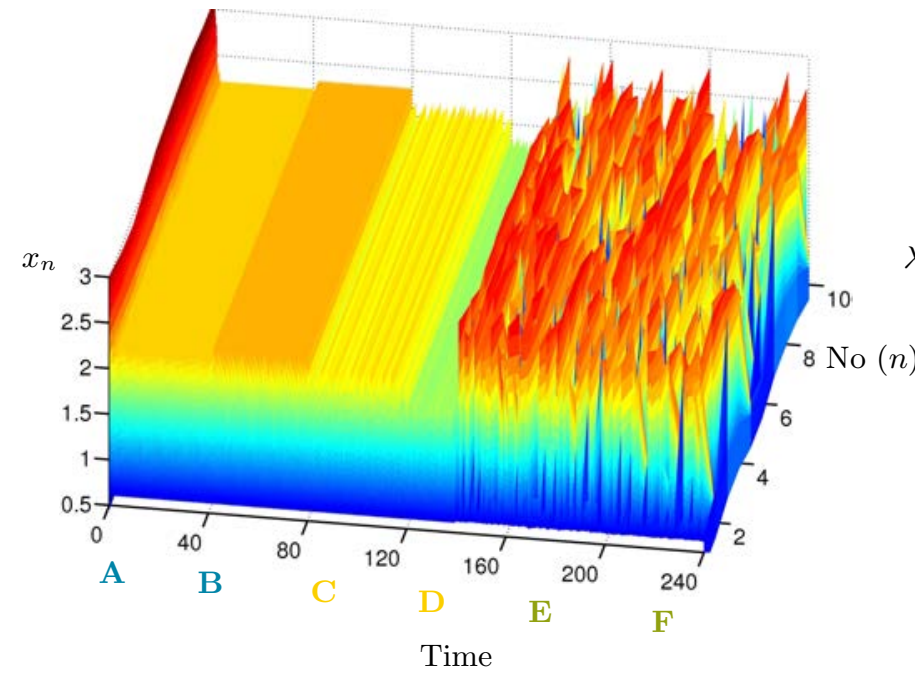

FIG. 22. Different states of the uncontrolled 1D BZ system $(n=10)$ for different values of the BZ bifurcation parameter $k_{f}$ (see table II). From state $\mathrm{C}$ to the right (in time), the BZ reaction is chaotic, at about $t \approx 140$ (D), the system becomes very complex chaotic.

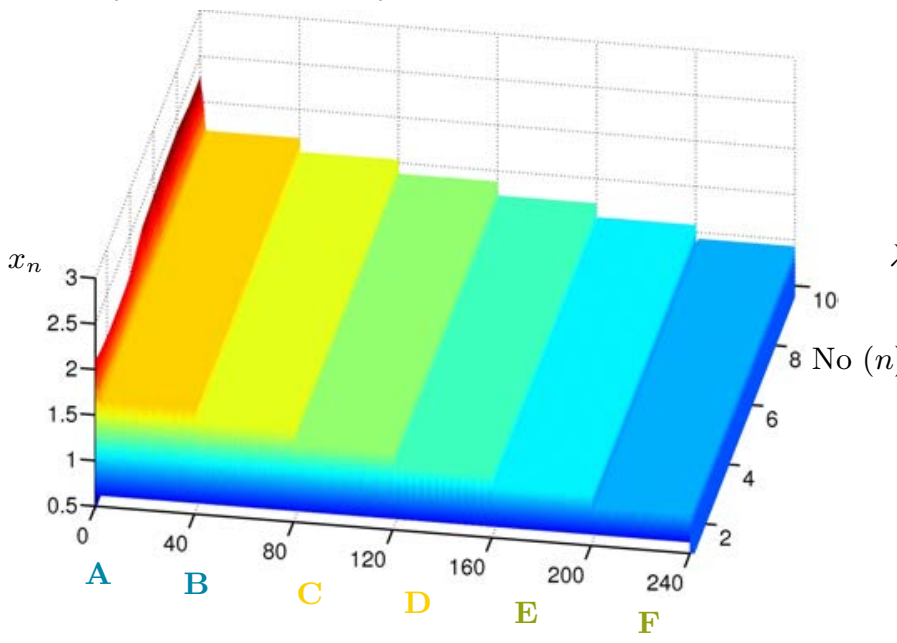

Time

FIG. 24. RCC of the $1 \mathrm{D}$ BZ system $(n=10)$ with control enabled throughout. Note that each bifurcation results in a somewhat different stable oscillation, which is reached almost instantaneously.

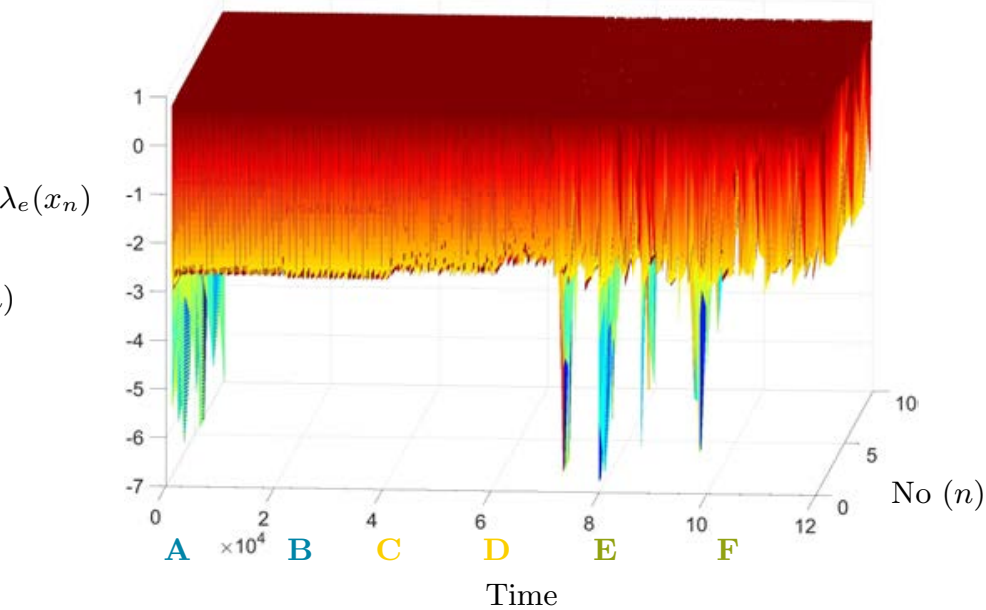

FIG. 23. Lyapunov estimates of the uncontrolled 1D BZ system of variables $x_{n}$, showing small positive estimates throughout, with wider variations in the complex chaotic domain.

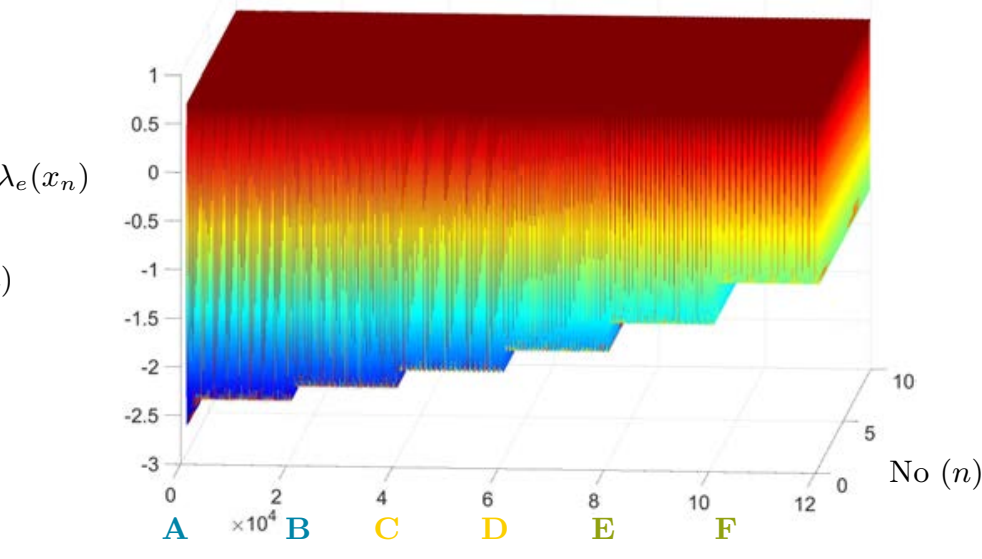

Time

FIG. 25. Lyapunov estimates of the RCC controlled 1D BZ system of the variable $x_{n}$, where the largest values are still small positive.

\section{DISCUSSION}

The rate control of chaotic systems is a relatively novel and effective method to stabilise unstable periodic orbits contained in chaotic systems. It does not require a priori knowledge of the UPOs contained in the system, however, it requires access to some of the system variables and the rate of change of some terms. Finding and stabilising different UPOs can easily be achieved experimentally, by changing the control parameters $\xi$, $\mu$, and $f$. Some experimentation with the control applied to different terms within the system, may be needed to get the best results. This implies that RCC may be employed as a generic black box control method, if an effective application point for the controller function can be found in the system.

Attempts to control different chaotic systems have been successful, but it appears that some terms in a set of equations are more effective at controlling the chaotic dynamics than others. This is more obvious in a relatively simple system, such as the Ikeda map [11], but in more complex dynamic systems this is not readily evident. A systematic analysis of the relative contributions of rate control of different system variables may be required to 
determine if this effect is due to the control method, or due to properties of the chaotic system itself. If rate control of different system variables indeed result in variable control of the chaotic system, this may give some clue to identifying which variables are more effective rate limiting than others. It may also be the case that the total control of the chaotic system is distributed over all rate variables in different proportions.

The application of the Rate Control of Chaos to different chaotic systems has been effective in every system attempted, on which more will be reported in subsequent publications. Although it is not expected to be universally applicable, due to the limitation of the lack of targeting, and the ability of the control to find suitable orbits, it has been effective in both maps and flows [11]. Furthermore, it has been shown that asymptotic stability is characteristic for the control method, but does not necessarily contained the system within the original basin of attraction, as is the case for the $\rho=28$ bifurcation parameter in the three dimensional Lorenz system. It may be argued that the constraints regarding an odd number of real characteristic multipliers greater than unity, which limits the applicability of the delay feedback control, also applies to RCC. However, the method allows the system to stabilise in different parts of the phase space by local adjustment, potentially avoiding this issue. Further analysis of the method will be pursued, but is limited due to the fact that the application of the control is determined by its ability to vary the local dynamics, which makes generalisation of the method more difficult. This also explains the difficulty of determining which variables should be controlled for any given systems, and where the control should be applied. On the other hand, empirical or evolutionary methods have been effective to determine points of control, which fits in with the biological interpretation of the method. The resulting controlled system has several interesting properties that may indicate the introduction of a localised hyperbolic equilibrium by the application of the control, such that the controlled system is equivalent to a topologically linearised system when it is controlled [29]. This requires the system to be exponentially stable, which is undetermined as yet.

The described method of rate limiting control can be argued to be similar to other rate controlling mechanisms such as enzymatic control of biochemical processes. In such systems, the flux of the resulting product is determined by the relative control of each of the enzymatic controlled steps [30]. Such a mechanism would avoid the occurrence of chaotic dynamics by preventing the system to become chaotic. A period doubling cascade, for example, would lead to a several stable controlled orbits, whenever the bifurcation parameter causes a change in the system, instead of becoming unstable. To demonstrate this in a biochemical setup would require further work in biochemical analysis, and possibly molecular biology, to explore the parameter space in which RCC of enzymatic processes would operate. This may then show a potential new interpretation of enzymatic control in controlling complex systems in biology. It is suggested that this mechanism can have evolved readily in biological systems, and would appear to be a natural way to eliminate undesirable chaos.

Lastly, the application of RCC to a spatiotemporal system demonstrates its ability to maintain control in perturbed systems. This opens the possibility to apply control to unstable perturbed systems that are normally not easy to control. For example, a system such as a wind turbine, perturbed chaotically by the wind, can then be more effectively controlled by RCC, instead of using linearised control methods, such as PID. Because RCC may remain enabled if the system is already stable, it will be effectively work unsupervised, and adjust the blades of the turbine to the localised changes to maintain stability of the system. This is currently under investigation, with some promising initial results.

\section{CONCLUSION}

The Rate Control of Chaos method of nonlinear control is capable of controlling a chaotic system into a stable state. It does this by making proportional changing to the rate of change of some of the terms in a set of equations. RCC is a feedforward type of control, which requires only the local state of the system to function effectively. If the system is perturbed by external input, noise, or neighbouring systems, RCC can compensate for this, maintaining stability. Furthermore, if the system goes through bifurcations, it is possible to stabilise the system into different orbits. This allows the control to be included in the system, independent of the need for control. If the system is already stable, RCC control can keep the stability, but may affect the specific periodic orbit into a somewhat different stable orbit. $\mathrm{RCC}$ is a true control of chaos method, only changing the dynamics of the system as needed, in a limited fashion. It is, therefore, unlikely to be successful in all possible control scenarios, however, due to its fast nonlinear nature, may perform well in most cases.

[1] E. Ott, C. Grebogi, and J. A. Yorke, Physical Review Letters 64, 1196 (1990).

[2] J. Starrett, Physical Review E 67, 1 (2003).

[3] K. Pyragas, Physics Letters A 170, 99 (1992). 
[4] K. Pyragas, Physics Letters A 206, 323 (1995).

[5] K. Pyragas, Physical Review Letters 86, 2265 (2001).

[6] J. E. Socolar, D. W. Sukow, and D. J. Gauthier, Physical Review E 50, 3245 (1994).

[7] H. Nakajima, Physics Letters A 232, 207 (1997).

[8] H. Nakajima, Physica D: Nonlinear Phenomena 111, 143 (1998).

[9] N. T. Crook and T. olde Scheper, Cybernetics and Systems 33, 341 (2002).

[10] H. G. Schuster, Handbook of Chaos Control, edited by H. G. Schuster (Wiley-VCH Verlag GmbH, Weinheim, FRG, 1999).

[11] T. olde Scheper, Biosystems 94, 145 (2008).

[12] A. Garfinkel, M. Spano, and W. Ditto, Science 257, 1230 (1992).

[13] L. Michaelis and M. L. Menten, Biochem Z 49, 333 (1913).

[14] G. Briggs and J. Haldane, Biochemical journal 19, 338 (1925).

[15] G. C. Brown, H. V. Westerhoff, and B. N. Kholodenko, Journal of theoretical biology 182, 389 (1996).

[16] L. A. . Segel and M. Slemrod, SIAM Review 31, 446 (1989).

[17] E. Ott, Chaos in Dynamical Systems (Cambridge University Press, 1993).

[18] T. Gao, Z. Chen, Q. Gu, and Z. Yuan, Chaos, Solitons \& Fractals 35, 390 (2008).

[19] W. Wu, Z. Chen, and Z. Yuan, Chaos, Solitons \& Fractals 39, 2340 (2009).

[20] H. Kantz, Physics Letters A 185, 77 (1994).

[21] R. Hoyle, Pattern Formation (Cambridge University Press, 2006).

[22] D. Auerbach, Physical Review Letters 72, 6 (1994).

[23] V. Petrov, S. Metens, P. Borckmans, and G. Dewel, Physical Review Letters 75 (1995).

[24] M. A. M. Henson, IEEE Control Systems Magazine , 54 (2006).

[25] Y. Nishiura, Physica D: Nonlinear Phenomena 150, 137 (2001).

[26] V. Petrov, V. Gaspar, J. Masere, and K. Showalter, Nature 361, 240 (1993).

[27] L. Györgyi and R. Field, The Journal of Physical Chemistry 95, 6594 (1991).

[28] L. Györgyi and R. J. Field, Nature 355, 808 (1992).

[29] A. Katok, Introduction to the modern theory of dynamical systems (Cambridge University Press, 1995).

[30] D. Fell, Understanding the control of metabolism. (Portland Press Ltd., 1997). 